\title{
Heterogeneous nucleation and microstructure formation: Steps towards a system and scale bridging understanding ${ }^{\star}$
}

\author{
H. Emmerich ${ }^{1}$, P. Virnau ${ }^{2}$, G. Wilde ${ }^{3}$, and R. Spatschek ${ }^{4}$ \\ 1 Chair for Materials and Process Simulation, Bayreuth University, Germany \\ 2 Institut für Physik, Johannes Gutenberg-Universität, Germany \\ 3 Institut für Materialphysik, Westfälische Wilhelms-Universität Münster, Germany \\ 4 Computational Materials Design Department, Max-Planck-Institut für Eisenforschung, \\ Düsseldorf, Germany
}

For 6 years from 2007-2013 the DFG has funded the scientific priority program 1296 with the above title. Its aim was to attain a fundamental understanding of the basic mechanisms underlying heterogeneous nucleation as well as the subsequent development of the nucleus into a specific heterogeneous microstructure. To that end a system- and method-spanning scientific approach was developed, in which the simplest types of model systems for heterogeneous crystalline orders, for pure metals, binary metal alloys, and colloids, were investigated in a comparative manner. Moreover they were assessed with complementary experimental as well as simulation techniques from the atomic to the mesoscale to obtain a comprehensive view across all relevant time and length scales, as depicted in Fig. 1. The figure illustrates the key idea of the priority program, namely to exploit a smearing of scales as given by the two complementary material systems for a most comprehensive assessment of the diverse kinetic, energetic and morphological features involved in heterogenous nucleation.

Some of the questions explored in detail in the context of the priority program are summarized in the following:

- Heterogeneous nucleation: What does a critical nucleation grain look like? Does the classical concept of a contact angle make sense for heterogeneous nucleation? Can claims be made about the dominant contributions to the nucleation barrier for heterogeneous nucleation, like they can be obtained from molecular simulations,

* During the course of the priority program one of its members, Prof. Dr. R. Bormann, died in a tragic accident. In his scientific work R. Bormann pushed with full enthusiasm particularly fundamental problems related to the phase transformation in metallic alloys, as they were also a central focus of this priority program. He supported the priority program from its initial stages: first as project leader, later he became president of University of Bayreuth. In that role he took responsibility in particular for fostering the frame for interdisciplinary scientific activities - a second link to the efforts and achievements in this priority program. We dedicate this overview article on the work in the priority program 1296 to the enthusiasm with which he engaged himself for both roles. 


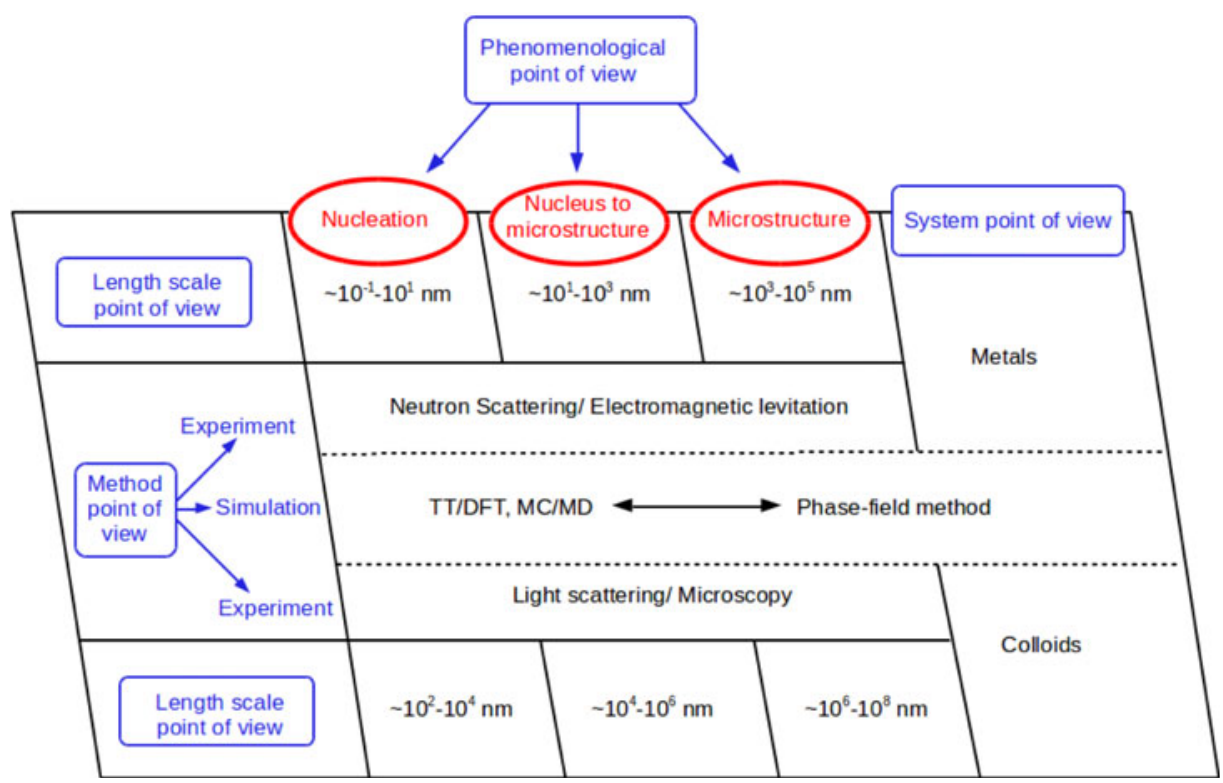

Fig. 1. Schematics of the interaction of different theoretical and experimental techniques to access heterogenous nucleation kinetics and dynamics most comprehensively over several time and length scales.

be reconciled with data gained via the phase-field method? What is the relation between interaction potentials and the relevant boundary energies?

- Transition from nucleus to microstructure: How does microstructure develop out of a nucleus in the interplay between crystallisation and segregation depending on the precise reference point in the phase diagram? How stable are those scenarios with respect to changes of that reference point? How well can these scenarios be reproduced via binary colloidal model systems?

- Microstructure development: What kind of consequences result from the new understanding of nucleation for the initial development of the microstructure? What kind of kinetic rules does the initial growth of the solidifying microstructure follow? Is it possible to identify universal conditions under which several microscopic morphologies of the same alloy composition are kinetically stable?

The priority program has been visible at the most relevant conferences and workshops of the participating communities all over the 6 years. This volume puts together the steps that could be taken beyond the classical nucleation theories and discusses critically open future directions. To that end it is divided into two parts devoted to the investigation of metals respectively colloids. Both parts contain research articles that present most recent new results as well as minireviews in which groups of complementary expertise jointly embed their achievements in the interdisciplinary research landscape driving the advances in the field.

In the following few paragraphs the key results for both material systems are presented in an overview. The presentation is divided in two sections on colloids and metals, respectively, due to a corresponding organisation of the work flow within the priority program. One guiding question beyond the concept of the priority program, namely, in how far the experimentally easier accessible colloids can serve as model systems for metals, is addressed inherently. 


\section{Colloids}

In the final funding period substantial progress within the focus group has been achieved by a number of successful collaborations between experiments and simulations and between theoretical groups which aimed at bridging the gap between phase field crystal methods, density functional theory and simulations at the particle level. Projects also benefitted from a vivid exchange of ideas and techniques between the focus groups, which allowed the projects partners to tackle colloidal and metallic systems with the same set of tools.

\section{1. $\mathrm{DFT} / \mathrm{PFC}$}

- In the projects of M. Oettel and T. Schilling (who was co-funded by INTER, Luxembourg) descriptions of hard-sphere crystals and crystal-fluid interfaces based on density functional theory (DFT) have been compared with a corresponding phase field crystal approach. It was shown that the order parameter of the phase-field crystal model is more consistent with a smeared density field, and that the phase field crystal model should not solely be interpreted as stemming directly from Taylor-expanded DFT.

- The liquid-solid interface in the hard sphere system is a prime example for the importance of entropy in the physics of the liquid-solid transition. In a collaboration between M. Oettel, J. Horbach, H. Löwen and S. Egelhaaf, a complete picture of this interface in terms of classical DFT theory was developed. Microscopic DFT predicts, e.g., a tension of $0.66 k_{B} T / \sigma^{2}$ with a small anisotropy and stiffnesses of $0.53 k_{B} T / \sigma^{2}$ for the (001) orientation and $1.03 k_{B} T / \sigma^{2}$ for (111). These results were corroborated by Monte Carlo simulations and compared with experimental findings.

- Classical DFT and dynamical DFT have also been applied in the project of H. Löwen and M. Schmiedeberg to study growth processes of hard discs on patterned substrates. A novel rhombic pre-ordering on a substrate with square symmetry was discovered, and by deploying dynamical DFT it was revealed that the growth process of the hard disk system on the square substrate is driven by compatibility waves.

- Additionally in the group of Ch. Holm computer simulation studies of a Lennard-Jones liquid confined in a narrow slit pore with tunable attractive walls were carried out. Main results related to that project were the finding that the behaviour of the Lennard-Jones liquid in a slit pore does not show crucial differences from the behaviour of the bulk while freezing unless the slit is narrow and that order parameters that characterize a system as a whole like radial distribution function or bond-order parameter cannot distinguish whether the system is homogeneous. As a consequence scaling of order parameters is necessary to check the homogeneity of the system and absence of defects.

\section{EXPERIMENTS}

- In the project of S. Egelhaaf heterogeneous nucleation of hard and soft spheres in the presence of flat and curved walls has been studied with confocal microscopy. For flat walls particles near the wall initially rearrange before crystal growth. During growth a depletion zone next to the progressing crystal-fluid interface can be observed. While flat walls lead to regular crystal structures, curved interfaces induce non-ideal crystal layering. These results have been corroborated by corresponding simulations in the project of $\mathrm{H}$. Löwen and M. Schmiedeberg. Curved interfaces have a somewhat lesser influence on systems consisting of soft spheres, which are able to counter-balance the deformations as established in a collaboration with W. Richtering. 
- Within the project of H.J. Schöpe heterogenous nucleation and microstructure evolution in one- and multi-component systems of various particle interactions were studied in real and reciprocal space using various time resolved optical techniques. For curved smooth substrates it was found that the nature of particle interaction highly modifies the process of nucleation and the microstructure formation. Further, the dependence of crystallization rates in heterogeneous nucleation on the structure of solid walls was investigated experimentally and by simulations in the projects of T. Schilling and M. Oettel. In the commensurate case, crystal growth was observed while in the incommensurate case crystallization proceeds via the classical nucleation and growth mechanism. At large lattice mismatches crystallization was blocked. A controlled addition of seeds into colloidal systems was also investigated in close analogy to inoculation in metallic systems. Here, the nucleation process determines the resulting microstructure, which could be modified over a wide range in width, shape and averaged crystal size.

- The main focus of a project by G. Maret and U. Gasser was the homogeneous and heterogeneous crystal nucleation on curved surfaces with radii of curvature, $\mathrm{R}$, in the range from 4 to 40 particle diameters. Heterogeneous crystal nucleation was suppressed for $R<10$ particle diameters. For $R>15$ particle diameters, fast crystal growth was observed similar to that on a flat wall, implying that the surface tension of the crystal on the seed surface depends on curvature; a result that goes beyond classical nucleation theory. Another central finding is related to the smallest precritical crystal nuclei which are found to be unaffected by curvature.

- An additional colloids related contribution to the priority program was the experimental work in the group of T. Palberg, which is documented independently from this volume. This group investigated in particular the influence of a variation of a colloidal system's potential on its heterogenous nucleation kinetics based on well understood homogeneous nucleation characteristics of the systems, with an outlook on a precise understanding of gravitation effects.

\section{SIMULATIONS}

- Through simulations, homogeneous nucleation in the hard sphere system has been shown to follow a two-step scenario, which is also seen in experimental results (collaboration of T. Schilling, M. Oettel and H. Schöpe). In a first step dense, amorphous clusters appear which act as precursors for nucleation of well-ordered crystals.

- In the project of P. Virnau and K. Binder the so called ensemble switch method to compute excess free energies of fluids and solids against planar walls was developed and applied to hard-sphere systems (which was studied by M. Oettel with DFT) as well as the Asakura-Oosawa model. For the latter, partial wetting with a contact angle of 70 degrees was observed and wall-attached droplets were found to be roughly sphere-cap shaped rather than facetted. In another collaboration with M. Oettel the Tolman hypothesis on the curvature dependence of the interfacial tension was carefully tested for several models. This length is negative for (liquid) droplets and about an order of magnitude smaller than the particle diameter. For the range of droplet sizes relevant for nucleation the next order quadratic correction was found to be more important.

\section{Metals}

Work on metallic systems during the third funding period has focused on nucleation and microstructure evolution in model systems for specific important aspects 
of the solidification process. Following directly from this approach, pure metals as well as selected alloy systems have been investigated that were carefully chosen to allow in-depth investigations concerning specific aspects of the nucleation and microstructure evolution processes. The close interlock between Theory/Simulation and Experiment has enabled substantial increase in understanding of the underlying processes on different time and length scales. The most important results obtained in the collaborative projects can be summarized as follows:

- Heterogeneous nucleation and microstructure formation during solidification of ternary $\mathrm{Al}-\mathrm{Cu}-\mathrm{Ni}$ alloys was studied by combining experimental work with thermodynamics and phase-field modeling (R. Schmid-Fetzer and H. Emmerich). The focus was on peritectic monovariant three-phase and invariant four-phase reactions. Peritectic solidification reactions and their associated nucleation and growth effects appear in many metallic systems but are less well understood than singlephase or eutectic solidification reactions. The ternary $\mathrm{Al}-\mathrm{Cu}-\mathrm{Ni}$ system is not only relevant for applications in aluminum alloys, furthermore it offers a range of peritectic-type and transition-type reactions in the Al-rich composition range to be studied as model examples. It is found that many experimentally observed features cannot be explained by simple Scheil calculations but are well understood by the improved multi-phase-field model. The advancements involve the application of the equilibrium parameters evaluated from the refined free energies of the phases. That enables a scale transcending understanding of these complex ternary solidification reactions, and allows to distinguish more precisely the precise nucleation kinetics in different types of binary and ternary peritectic systems.

- Heterogeneous nucleation and growth have been studied experimentally, by modeling and phase field simulations in Ti-Al alloys (M. Apel, R. Günther and F. Pyczak). Heterogeneous nucleation and thus grain refinement was achieved by boron additions, either elementary boron or by the inoculation of the melt with $\mathrm{TiB}_{2}$-particles. The grain refinement has been analyzed for different boron levels, Al compositions, i.e. for peritectic and hyperperitectic alloys, as well as for different cooling rates. In terms of modeling, lattice mismatch models have been developed which allow identifying those $\mathrm{TiB}_{2}$-lattice planes which show the highest nucleation potency for both, alpha-Ti and beta-Ti phase. The standard model for nucleation and free growth does not lead to a satisfying match with the experimental results but much better agreement can be obtained by taking into account the facetted particle morphology which leads to a smaller effective size for the determination of the growth barrier. Phase field models have been quantitatively adapted to the simulation of the peritectic solidification in $\mathrm{Ti}-\mathrm{Al}$ including boron. Simulations could be used to visualize different nucleation and growth scenarios, e.g. it could be shown that the fractions of the peritectic and properitectic phases at the end of solidification strongly depend on the location of the nucleation events, i.e. whether nucleation occurs on the properitectic phase or on $\mathrm{TiB}_{2}$-particles in the interdendritic region.

- Crystal nucleation at deep undercooling in one-component metals that crystallize in the fcc lattice has been investigated using a combination of differential thermal analysis experiments and Monte Carlo (MC) simulations (G. Wilde and J. Horbach). Applying novel methodologies developed within this project, nucleation rates of $\mathrm{Au}, \mathrm{Cu}, \mathrm{Ni}$ and $\mathrm{Co}$ could be measured over eight orders of magnitude, allowing for the first direct comparison between results obtained in simulation (on $\mathrm{Ni}$ ) and experiment. Additionally, free energy nucleation barriers were extracted using an ansatz obtained in the framework of classical nucleation theory (CNT). The latter approach is rationalized by MC simulations that directly yield estimates for the temperature dependence of $\mathrm{G}^{*}$. The values of $\mathrm{G}^{*}$, as determined from the simulation, are in very good agreement with those extracted from the experiments. The simulations indicate that in the range where experiments are available the 
corrections to CNT are relatively small, thus justifying the application of CNT. The observed nucleation mechanism was found to be either identical or at least highly similar across the investigated fcc metals and independent on purity and sample volume, indicating that homogeneous nucleation is a distinct possibility. Assuming homogeneous nucleation, a linear dependence of the interfacial free energy on the temperature was found as it is to be expected if the interfacial free energy is dominated by the contribution of the entropy. The experimentally determined interfacial free energy is $\sigma(T)=0.58 k_{B} T / a^{2}$, which is in good agreement with the results from atomistic simulations but at odds with the value predicted in the so-called negentropic model.

- In the group of B. Nestler, the structural and kinetic properties of early stage solidification patterns have been studied by computations based on continuum and atomistic methods. In particular, phase-field and molecular dynamics simulations have been conducted on the atomic scale and the resulting growth structures have been compared. Furthermore, atomistic methods such as phase-field crystal and molecular dynamics approaches operating on the atomistic scale have been combined with phase-field models on mesoscopic length scales to investigate the influence of nucleation characteristics on the growth of dendritic and eutectic microstructures. To establish a computational framework capable to bridge the scales, various techniques such as successive rescaling algorithms and symmetry constructions to optimize the computational efficiency have been developed and applied to model large scale dendritic growth. New phase-field crystal methods for multicomponent systems have been formulated incorporating energy formulations proposed by density functional theories to predict the phase formation in binary and ternary eutectic systems. The new atomistic approaches are expected to provide essential material data such as phase boundary properties and kinetic properties required for an integrated transfer into the well-established microstructures simulations.

- During the last funding period of SPP 1296, scaling laws for the pattern formation in three phase binary alloys, i.e. eutectic, monotectic and syntectic systems, using boundary integral technique and phase-field simulations have been developed in the group of R. Spatschek and H. Müller-Krumbhaar. The growth velocity, pattern scales, morphology and stability have been predicted for the diffusion limited regime. They also addressed problems where elasticity and plasticity are of major importance, including a coupling to phase field descriptions, which is a central element in the entire priority program. The influence of misfit stresses on the equilibrium shape of a crystal when it loses the coherency with its supporting substrate during heterogeneous nucleation has been studied. As a result, the different regimes of nucleation in a confined geometry between two parallel misfitting substrates as a function of the thermodynamic driving force and the degree of confinement have been classified. The combination of misfit stresses and plastic deformations can lead to extended defects in nanoscopic objects. Concerning this, the influence of the torsion in an Eshelby twisted nanowire on the development of the Rayleigh-Plateau instability has been investigated, which can trigger the degradation of nanowires. Apart from this, the role of grain boundary premelting has been studied using amplitude equations descriptions, phase field crystal modeling and molecular dynamics simulations. It is found that above a critical misorientation localized melting in the grain boundary can occur already below the bulk melting point; a comparison of the predictions to molecular dynamics simulations has been performed for bcc iron. The model descriptions also play an important role for the bridging aspects of the priority program, as they naturally connect to phase field crystal and density functional theoretical approaches used for colloidal systems. 
- In an additional project by D. Herlach attached to the priority program in its first phase the phase behaviour of silica suspensions consisting of silica particles of average size $77 \mathrm{~nm}-84 \mathrm{~nm}$, respectively, dispersed in water of high purity were investigated. The phase behaviour was studied leading to the construction of a phase diagram, in which the regions of existence of liquid and solid bcc phase are shown in dependence on the particle number densities and the concentration of $\mathrm{NaOH}$.

The success of the priority program can be seen on the one hand in its wide-ranging interdisciplinary visibility via organized symposia and invited talks at the most relevant conferences in the materials science and computer simulation field, as at the

- TMS Annual Meeting 2009, 2010, 2013

- Euromat 2009, 2011, 2013

- Material Science and Engineering 2010

- DPG Frühjahrstagung 2011

- MRS Spring Meeting 2011, 2012

- 85th ACS Colloids and Surfaces Symposium 2011

- 3rd International Conference on Ferromagnetic Shape Memory Alloys 2011

- 18th American Conference on Crystal Growth and Expitaxy

- MRS Fall Meeting 2011

- 8th European Solid Mechanics Conference (ESMC) 2012

- Materials Science Engineering 2012

- Multiscale Materials Modeling (MMM) 2012

- 19th American Conference on Crystal Growth and Epitaxy 2013.

On the other hand the scientific advances to which it could contribute have been documented in a large number of well visible papers in renowned journals of the field:

\section{References}

1. S. Praetorius, A. Voigt, R. Wittkowski, H. Löwen, Phys. Rev. E 87, 052406 (2013)

2. S. van Teeffelen, C.V. Achim, H. Löwen, Phys. Rev. E 87, 022306 (2013)

3. V. Heinonen, A. Mijailovic, C.V. Achim, T. Ala-Nissila, R.E. Rozas, J. Horbach, H. Löwen, J. Chem. Phys. 138, 044705 (2013)

4. T. Neuhaus, M. Schmiedeberg, H. Löwen, New J. Phys. 15, 073013 (2013)

5. T. Neuhaus, M. Marechal, M. Schmiedeberg, H. Löwen, Phys. Rev. Lett. 110, 118301 (2013)

6. A. Reinmüller, H.J. Schöpe, T. Palberg, Langmuir 29, 1738 (2013)

7. A. Reinmüller, H.J. Schöpe, T. Palberg, Rev. Sci. Instr. 84, 063907 (2013)

8. R. Spatschek, B. Eidel, Int. J. Solids Struct. 50, 2424 (2013)

9. A. Adland, A. Karma, R. Spatschek, D. Buta, M. Asta, Phys. Rev. B 87, 024110 (2013)

10. G. Boussinot, Sur. Sci. 607, L1 (2013)

11. S. Praetorius, A. Voigt, R. Wittkowski, H. Löwen, Phys. Rev. E 87, 052406 (2013)

12. F. Ziese, G. Maret, U. Gasser, J. Phys.: Condens. Matter 25, 375105 (2013)

13. M. Anwar, F. Turci, T. Schilling, J. Chem. Phys. 139, 214904 (2013)

14. S. Dorosz, T. Schilling, J. Chem. Phys. 139, 124508 (2013)

15. R. Benjamin, J. Horbach, J. Chem. Phys. 139, 084705 (2013)

16. F. Schmitz, P. Virnau, K. Binder, Phys. Rev. E 87, 053302, 1 (2013)

17. K. Binder, B.J. Block, P. Virnau, A. Tröster, Am. J. Phys. 80, 1099 (2013)

18. A. Winkler, A. Statt, P. Virnau, K. Binder, Phys. Rev. E 87, 032307, 1-13 (2013)

19. A. Carre, B. Böttger, M. Apel, J. Crystal Growth 380, 5-13 (2013)

20. M.H. Yamani, M. Oettel, Phys. Rev. E 88, 022301 (2013)

21. M. Berghoff, M. Selzer, B. Nestler, The Scientific World Journal, Vol. 2013, Article ID $564272(2013)$ 
22. C. Hüter, G. Boussinot, E.A. Brener, R. Spatschek, Phys. Rev. E 86, 021603 (2012)

23. J. Kundin, R. Siquieri, H. Emmerich, Physica D 243, 116127 (2012)

24. M. Oettel, S. Dorosz, M. Berghoff, B. Nestler, T. Schilling, Phys. Rev. E 86, 021404 (2012)

25. S. Dorosz, T. Schilling, J. Chem. Phys. 136, issue 4 (2012)

26. M.A. Choudhary, J. Kundin, H. Emmerich, Philosophical Magazine Letters 92, 451$458(2012)$

27. R. Benjamin, J. Horbach, J. Chem. Phys. 137, 044707 (2012)

28. R. Benjamin, J. Horbach, Erratum of Paper, J. Chem. Phys. 139, 039901 (2013)

29. M. Guerdane, H. Teichler, B. Nestler, Phys. Rev. Lett. 110, 086105 (2013)

30. M. Guerdane, H. Teichler, B. Nestler, Phys. Rev. Lett. 086105-1-086105-5

31. A. Reinmüller, E.C. Oguz, R. Messina, H. Löwen, H.J. Schöpe T. Palberg, J. Chem. Phys. 136, 164505 (2012)

32. A. Lederer, H.J. Schöpe, Phys. Rev. E 85, 031401 (2012)

33. A. Engelbrecht, H.J. Schöpe, Soft Matter 8, 11034 (2012)

34. T. Palberg, M. Maaroufi, A. Stipp, H.J. Schöpe, J. Chem. Phys. 137, 094906 (2012)

35. R. Beyer, S. Iacopini, T. Palberg, H.J. Schöpe, J. Chem. Phys. 136, 234906 (2012)

36. A. Härtel, M. Oettel, R.E. Rozas, S.U. Egelhaaf, J. Horbach, H. Löwen, Phys. Rev. Lett. 108, $226101(2012)$

37. A. Tröster, M. Oettel, B.J. Block, P. Virnau, K. Binder, J. Chem. Phys. 136, 064709 (2012)

38. D. Deb, A. Winkler, P. Virnau, K. Binder, J. Chem. Phys. 136, 134710 (2012)

39. D. Deb, D. Wilms, A. Winkler, P. Virnau, K. Binder, Int. J. Mod. Phys. C 23, 1240011 (2012)

40. C. Gögelein, D. Wagner, F. Cardinaux, G. Nägele, S.U. Egelhaaf, J. Chem. Phys. 136, $015102(2012)$

41. R. Roth, K. Mecke, M. Oettel, J. Chem. Phys. (Comm.) 136, 081101 (2012)

42. M. Oettel, J. Phys.: Condens. Matter 24, 464124 (2012)

43. W. Guo, R. Spatschek, I. Steinbach, Physica D 240, 382 (2011)

44. M.A. Choudhary, D. Li, H. Emmerich, H. Löwen, J. Phys.: Condens. Matter. 23, 265005 (2011)

45. G. Boussinot, C. Hüter, E.A. Brener, Phys. Rev. E 83, 020601 (2011)

46. D. Deb, A. Winkler, M.H. Yamani, M. Oettel, P. Virnau K. Binder, J. Chem. Phys. 134, 214706 (2011)

47. A. Engelbrecht, R. Meneses, H.J. Schöpe, Soft Matter 7, 5685 (2011)

48. M. Franke, A. Lederer, H.J. Schöpe, Soft Matter 7, 11267 (2011)

49. K. Sandomirski, E. Allahyarov, H. Löwen, S.U. Egelhaaf, Soft Matter 7, 8050 (2011)

50. J. Bokeloh, R.E. Rozas, J. Horbach, G. Wilde, Phys. Rev. Lett. 107, 145701 (2011)

51. R. Spatschek, E. Brener, A. Karma, Philosophical Mag. 91, 75 (2011)

52. H.J. Schöpe, P. Wette, Phys. Rev. E 83, 051405 (2011)

53. N. Gribova, A. Arnold, T. Schilling, C. Holm, J. Chem. Phys. 135, 054514 (2011)

54. C. Hüter, G. Boussinot, E.A. Brener, D.E. Temkin, Phys. Rev. E 83, 050601(R) (2011)

55. M. Fleck, D. Pilipenko, R. Spatschek, E.A. Brener, Phys. Rev. E 83, 046213 (2011)

56. J. Kundin, H.-L. Chen, H. Emmerich, R. Schmid-Fetzer, Eur. Phys. J. Plus 126, 96 (2011)

57. J. Kundin, R. Siquieri, Physica D 240, 459 (2011)

58. R.E. Rozas, J. Horbach, Europhys. Lett. 93, 26006 (2011)

59. B. Nestler, A. Choudhury, Curr. Opinion Solid State Mater. Sci. 15, 93 (2011)

60. S. Seiffert, J. Dubbert, W. Richtering, D.A. Weitz, Lab on a Chip 11, 966 (2011)

61. H.-L. Chen, E. Doernberg, P. Svoboda, R. Schmid-Fetzer, Thermochim. Acta 512, 189 (2011)

62. R. Spatschek, A. Karma, Phys. Rev. B 81, 214201 (2010)

63. H. Löwen, J. Phys: Condens. Matter. 22, 364105 (2010)

64. P. Wette, I. Klassen, D. Holland-Moritz, D.M. Herlach, H.J. Schöpe, N. Lorenz, H. Reiber, T. Palberg, S.V. Roth, J. Chem. Phys. 132, 131102 (2010)

65. R. Wittkowski, H. Löwen, H.R. Brand, Phys. Rev. E 82, 031708 (2010) 
66. A. Engelbrecht, H.J. Schöpe, Crystal Growth Design 10, 2258 (2010)

67. M. Oettel, S. Görig, A. Härtel, H. Löwen, M. Radu, T. Schilling, Phys. Rev. E 82, $051404(2010)$

68. D. Gosslar, R. Günther, U. Hecht, C. Hartig, R. Bormann, Acta Materialia 58, 6744 (2010)

69. N.J. Lorenz, T. Palberg, J. Chem. Phys. 133, 104501 (2010)

70. C. Hartig, R. Günther, D. Gosslar, R. Bormann, Int. J. Mater. Res. 101, 572 (2010)

71. N. Wang, R. Spatschek, A. Karma, Phys. Rev. E 81, 051601 (2010)

72. A. Stipp, H.J. Schöpe, T. Palberg, T. Eckert, R. Biehl, E. Bartsch, Phys. Rev. E 81, 051401 (2010)

73. T. Schilling, H.J. Schöpe, M. Oettel, G. Opletal, I. Snook, Phys. Rev. Lett. 105, 025701 (2010)

74. A. Härtel, R. Blaak, H. Löwen, Phys. Rev. E 81, 051703 (2010)

75. A. Reinmüller, H.J. Schöpe, T. Palberg, Soft Matter 6, 5312 (2010)

76. M. Fleck, C. Hüter, D. Pilipenko, R. Spatschek, E.A. Brener, Phil. Mag. 90, 265 (2010)

77. D.M. Herlach, I. Klassen, P. Wette, D. Holland-Moritz, J. Phys. Condens. Matter 22, $153101(2010)$

78. T. Zykova-Timan, J. Horbach, K. Binder, J. Chem. Phys. 133, 014705 (2010)

79. B.J. Block, S. Das, S.K. Das, M. Oettel, K. Binder, J. Chem. Phys. 133, 154702 (2010)

80. B. Nestler, D. Danilov, H. Guerdane, H. Teichler, J. Phys. D: Appl. Phys. 42, 015310 (2009)

81. P. Wette, I. Klassen, D. Holland-Moritz, T. Palberg, S.V. Roth, D.M. Herlach, Phys. Rev. E 79, 010501 (2009)

82. P. Wette, A. Engelbrecht, R. Salh, I. Klassen, D. Menke, D.M. Herlach, S.V. Roth, H.J. Schöpe, J. Phys.: Condens. Matter 21, 464115 (2009)

83. T. Zykova-Timan, R.E. Rozas, J. Horbach, K. Binder, J. Phys.: Condens. Matter 21, $464102(2009)$

84. S. Iacopini, T. Palberg, H.J. Schöpe, J. Chem. Phys. 130, 084502 (2009)

85. P. Espaol, H. Löwen, J. Chem. Phys. 131, 244101 (2009)

86. S. van Teeffelen, R. Backofen, A. Voigt, H. Löwen, Phys. Rev. E 79, 051404 (2009)

87. P. Wette, H.J. Schöpe, T. Palberg, Phys. Rev. E 80, 021407 (2009)

88. D. Gosslar, C. Hartig, R. Günther, U. Hecht, R. Bormann, J. Phys. 21, 464111 (2009)

89. G. Wilde, C. Santhaweesuk, J.L. Sebright, J. Bokeloh, J.H. Perepezko, J. Phys.: Condens. Matter 21, 464113 (2009)

90. B. Nestler, M. Selzer, M. Jainta, Fachz. Werkstoffe Fertigung 25800, 37 (2009)

91. D. Winter, P. Virnau, K. Binder, Phys. Rev. Lett. 103, 225703 (2009)

92. R. Siquieri, E. Doernberg, H. Emmerich, R. Schmid-Fetzer, J. Phys.: Condens. Matter 21, $164112(2009)$

93. E. Villanova-Vidal, T. Palberg, H.J. Schöpe, H. Löwen, Philosophical Mag. 89, 1695 (2009)

94. E.A. Brener, G. Boussinot, C. Hüter, M. Fleck, D. Pilipenko, R. Spatschek, D.E. Temkin, J. Phys.: Condens. Matter 21, 464106 (2009)

95. N. Lorenz, H.J. Schöpe, T. Palberg, J. Chem. Phys. 131, 134501 (2009)

96. N.J. Lorenz, H.J. Schöpe, H. Reiber, T. Palberg, P. Wette, I. Klassen, D. HollandMoritz, D. Herlach, T. Okubo, J. Phys.: Condens. Matter 21, 464116 (2009)

97. S. Iacopini, T. Palberg, H.J. Schöpe, Phys. Rev. E 79, 010601 (R) (2009)

98. J. Schmitz, J. Brillo, I. Egry, R. Schmid-Fetzer, Int. J. Mater. Res. 100, 1529 (2009)

99. L. Assoud, F. Ebert, P. Keim, R. Messina, G. Maret, H. Löwen, Phys. Rev. Lett. 102, 238301 (2009)

100. T. Palberg, A. Stipp, E. Bartsch, Phys. Rev. Lett. 102, 038302 (2009)

101. D. Gosslar, C. Hartig, R. Günther, U. Hecht, R. Bormann, J. Phys.: Condens. Matter 58, (2009)

102. R. Siquieri, H. Emmerich, J. Phys.: Cond. Matter 21, 464105/1-464105/5 (2009)

103. R. Siquieri, H. Emmerich, Steel Res. 1, 9 (2009)

104. J. Horbach, R.E. Rozas, T. Unruh, A. Meyer, Phys. Rev. B 80, 212203 (2009) 
105. J. Eiken, M. Apel, V. Witusiewicz, J. Zollinger, U. Hecht, J. Phys.: Condens. Matter 21, $464104(2009)$

106. I. Maltsev, A. Mirzoev, D. Danilov, B. Nestler, Modelling Simul. Mater. Sci. Eng. 17 (2009)

107. M. Schrader, P. Virnau, D. Winter, T. Zykova-Timan, K. Binder, Eur. Phys. J. Special Topics 177, 103 (2009)

108. D. Winter, P. Virnau, K. Binder, J. Phys.: Condens. Matter 21, 464118 (2009)

109. H. Garcke, B. Nestler, B. Stinner, F. Wendler, Math. Models Meth. Appl. Sci. 18, 1347 (2008)

110. S. van Teeffelen, C.N. Likos, H. Löwen, Phys. Rev. Lett. 100, 108302 (2008)

111. N. Lorenz, J. Liu, T. Palberg, Coll. Surf. 319(1-3), 109 (2008)

112. B. Nestler, F. Wendler, M. Selzer, B. Stinner, H. Garcke, Phys. Rev. E 78, 011604-1011604-7 (2008)

113. T. Palberg, N. Lorenz, H.J. Schöpe, P. Wette, I. Klassen, D. Holland-Moritz, D.M. Herlach (Wiley-VCH, 2008), p. 185

114. W.A. Oates, S.-L. Chen, W. Cao, F. Zhang, Y.A. Chang, L. Bencze, E. Doernberg, R. Schmid-Fetzer, Acta Mater. 56, 5255 (2008)

115. E.A. Brener, D.E. Temkin, Acta Mater. 56, 2290 (2008)

116. C. Gugenberger, R. Spatschek, K. Kassner, Phys. Rev. E 78, 016703 (2008)

117. D. Pilipenko, E. A. Brener, C. Hüter, Phys. Rev. E 78, 060603 (2008)

118. R. Spatschek, E. A. Brener, D. Pilipenko, Phys. Rev. Lett. 101, 205501 (2008)

119. U. Hecht, V. Witusiewicz, A. Drevermann, J. Zollinger, Intermetallics 16, 969 (2008) 